\title{
Diversidad florística y captura de carbono en robledales y pasturas con árboles en Santa Isabel, Tolima, Colombia
}

\section{Milena A. Segura-Madrigal ${ }^{1}$, Hernán J. Andrade C. ${ }^{2 *} \&$ Erika Sierra Ramírez ${ }^{3}$}

1. Grupo de Investigación PROECUT, Universidad del Tolima, Facultad de Ingeniería Forestal, Tolima, Colombia; masegura@ut.edu.co

2. Grupo de Investigación PROECUT, Universidad del Tolima, Facultad de Ingeniería Agronómica, Tolima, Colombia; hjandrade@ut.edu.co

3. Grupo de Investigación PROECUT, Universidad del Tolima, Facultad de Ingeniería Forestal, Tolima, Colombia; esierrar@ut.edu.co

* Correspondencia

Recibido 05-VI-2019. Corregido 04-IX-2019. Aceptado 13-II-2020.

\begin{abstract}
Floristic diversity and carbon capture in oak groves and pastures with trees in Santa Isabel, Tolima, Colombia. Introduction: The accelerated transformation of land uses in the Colombian Andes has affected diversity and the provision of ecosystem services. Objective: The structure and floristic composition and the estimation of the carbon stored in biomass of intervened primary forests and pastures with trees in Santa Isabel, Tolima, was described. Methods: In each system, five sampling plots were established: rectangular of $1000 \mathrm{~m}^{2}$ each in forests and circular of $707 \mathrm{~m}^{2}$ in pastures to measure all individuals with a diameter of $1.30 \mathrm{~m}$ in height, $\geq 10 \mathrm{~cm}$. The biomass was estimated from multi-species allometric models and a generic model in the case of below-ground biomass. Results: In the forest, an average abundance of $642 \pm 125$ individuals / ha was recorded, finding 25 families, 39 genera, 43 species and morphospecies in 0.5 ha of sampling. Quercus humboldtii and Ladenbergia macrocarpa were the species with the highest Importance Value Index (IVI $=32.5$ and 11.2, respectively) in the forests. In pastures, $175.0 \pm 21.3$ individuals / ha were found, belonging to 8 families, 9 genera, 10 species and morphospecies in 0.35 ha of sampling. Weinmannia pubescens and Eugenia spp. had the greatest ecological importance (19.5 and $17.6 \%$, respectively) in pastures. The forest stored an average of $125.0 \pm 30.0 \mathrm{t} \mathrm{C} / \mathrm{ha}$, compared to $18.4 \pm 1.8 \mathrm{t} \mathrm{C} / \mathrm{ha}$ in pastures. Conclusions: These findings show high carbon storage capacity in these land uses, which contributes to maintain or decrease the concentration of greenhouse gases in the atmosphere, and therefore, are a benchmark that allows to develop conservation strategies, with a view to mitigate climate change.
\end{abstract}

Key word: carbon storage, biomass, andean forest, diversity, Quercus humboldtii.

Segura-Madrigal, M.A., Andrade C., H.J., \& Sierra Ramírez, E. (2020). Diversidad florística y captura de carbono en robledales y pasturas con árboles en Santa Isabel, Tolima, Colombia. Revista de Biología Tropical, 68(2), 383-393.

El departamento del Tolima presenta una alta heterogeneidad en zonas de vida, su altitud va desde los 500 hasta los $5200 \mathrm{~m}$, lo que permite una alta diversidad florística (Campo, 2010; Fernández, Bernate, \& Melo, 2013). No obstante, la ampliación de cultivos, plantaciones y la ganadería extensiva están causando un desequilibrio en los paisajes de la región (Galindo, Betancur, \& Cadena, 2003). En particular, la transformación del cambio de uso del suelo en los ecosistemas andinos acelera la problemática ambiental comprometiendo la permanencia de 
los bosques y disminuyendo la provisión de servicios ecosistémicos (Mathez, Peralvo, \& Báez, 2017; IDEAM, 2017a).

La zona andina colombiana, localizada entre los 750 y los $3450 \mathrm{~m}$, se caracteriza por encontrar especies como el roble (Quercus humboldtii Bonpl.), cuyas aglomeraciones se denominan robledales, los cuales ofertan amplios servicios ecosistémicos (Martínez et al., 2017). Dentro de estos servicios se destaca la provisión de madera, belleza escénica, la protección del recurso hídrico, conservación de suelos, captura de carbono, regulación de los gases de efecto invernadero (Avella \& Cárdenas, 2010), aquellos derivados de la conservación de la diversidad (Pujade, Rodríguez, \& Caicedo, 2015) y la provisión de productos forestales no maderables tal como preparados de medicina tradicional, alimentos y bebidas, artículos de limpieza y envoltura para alimentos (Potosí, Villalba, \& Arboleda, 2017), que dependen, en gran medida de las decisiones e intervenciones de las familias productoras en sus fincas y del cumplimiento de las políticas nacionales (Andrade, Segura, \& Sierra, 2017).

A pesar que Quercus humboldtii Bonpl., domina ecológicamente grandes extensiones de tierra en el territorio nacional, sus áreas son deforestadas debido principalmente a la explotación de madera y la expansión de la frontera agropecuaria, lo cual ha causado un moderado riesgo de extinción o deterioro poblacional a mediano plazo. Esta especie es catalogada como vulnerable en el libro rojo de las plantas de Colombia (Cárdenas \& Salinas, 2006). Además, su aprovechamiento está en veda en todo el territorio colombiano (Ministerio de Ambiente Vivienda y Desarrollo Territorial, 2006).

Además, el sector agropecuario extensivo propicia la deforestación y degradación de los bosques andinos, lo cual constituye un emisor de gases de efecto invernadero (GEI) por el consumo de fertilizantes, descomposición anaerobia y producción de metano (Jiménez, González, \& Pazmiño, 2019). El Departamento Administrativo Nacional de Estadística, DANE (2014) ha estimado en 43.1 millones de ha destinadas al uso agropecuario, lo cual ha contribuido con el $26 \%$ de las $258.8 \mathrm{Mt}$ de $\mathrm{CO}_{2}$ del inventario de GEI en Colombia (IDEAM, 2016). Por ende, es indispensable recopilar información de la diversidad y estructura de estos ecosistemas, que llene vacíos de conocimiento para mejorar la educación ambiental, la aplicación de estrategias de mitigación del cambio climático y se prioricen futuras investigaciones que integren la inter y transdisciplinariedad para la conservación y gobernanza sostenible de estos paisajes (Mathez et al., 2017).

En esta perspectiva, este trabajo se constituye en un estudio base para evaluar el estado actual, composición florística y la captura de carbono en bosques del municipio de Santa Isabel, Tolima, Colombia. Los resultados permitirán el diseño de estrategias de conservación en estos ecosistemas andinos.

\section{MATERIALES Y MÉTODOS}

Área de estudio: El estudio se realizó en el Municipio de Santa Isabel, en la Cordillera Central colombiana, en la subregión de los nevados, al norte del departamento del Tolima

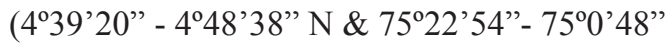
$\mathrm{W}) ; 2400 \mathrm{~m}$ de altitud. El área tiene una temperatura promedio de $16{ }^{\circ} \mathrm{C}$, con precipitación media anual de $1900 \mathrm{~mm}$ (IDEAM, 2017b), clasificado como Bosque Húmedo Montano Bajo (bh-MB), con algunas áreas dominadas por roble (Quercus humboldtii) (Holdridge, 1978; Cortolima, 2008). La topografía del terreno presenta pendientes superiores al $70 \%$, con facilidad de drenaje, suelos profundos y altamente susceptibles a la erosión (Rojas, Andrade, \& Segura, 2018).

El municipio contaría con 6301 habitantes para el 2019, según las proyecciones del DANE (2005) y su economía se basa principalmente en el sector agropecuario con productos como la arveja, papa, café, yuca, mora, tomate de árbol, lulo, leche y sus derivados, además, cuenta con áreas boscosas, humedales y la zona amortiguadora al Parque Natural de los Nevados (Consejo Municipal de Santa Isabel, 2016). 
Diseño de muestreo: Los sitios de muestreo se ubicaron en las veredas La Yuca y Buena Vista del municipio, entre los 2500 y los 2800 m.s.n.m. de altitud. Se empleó un diseño completamente a azar con dos tratamientos: 1) bosques primarios intervenidos, 2) y pasturas con árboles dispersos. Se denomina bosque primario intervenido a un bosque nativo que ha sido afectado por las actividades humanas pero que estas no causas efectos significativos en sus procesos ecológicos (FAO, 2010) y las pasturas tienen la particularidad de combinar árboles con animales dentro de un lote (FAO, 1999).

En cada cobertura de uso del suelo se realizaron cinco repeticiones. En cada repetición, se estableció una parcela permanente de muestreo (PPM) rectangular de $1000 \mathrm{~m}^{2}$ en bosques y circulares de $707 \mathrm{~m}^{2}$ en las pasturas arboladas. Se midió la altura total (ht) y el diámetro a $1.30 \mathrm{~m}$ de altura (dap) de los individuos con $\geq 10 \mathrm{~cm}$ de dap. La identificación taxonómica fue realizada en campo, con ayuda de personal local, y confirmada en el Herbario Toli de la Universidad del Tolima.

Análisis de estructura horizontal: $\mathrm{Se}$ estimó la riqueza, abundancia y la estructura horizontal (distribución diamétrica) en cada uso del suelo. Se cuantificó el índice de valor de importancia (IVI), el cual tiene en cuenta los valores relativos de la abundancia, frecuencia y dominancia de las especies. La caracterización de la diversidad de especies se estimó con base en los índices de equidad o diversidad de
Shannon-Wiener y de dominancia de Simpson (Moreno, 2001; Moreno, Barragán, Pineda, \& Pavón, 2011), calculado con el programa Past versión 3.16 (Hammer, Harper, \& Ryan, 2007).

Estimación de biomasa y carbono: Se estimó la biomasa de todos los individuos encontrados en las parcelas permanentes de cada uso del suelo. La biomasa arriba del suelo se estimó con un modelo local desarrollado por Lerma y Orjuela (2014) que incluye a Weinmannia auriculata, Miconia sp. y Baccharis sp. En los árboles con diámetros superiores a $60 \mathrm{~cm}$ se usó el modelo multi-especie recomendado por Álvarez et al. (2012) para bosques montanos de Colombia y para $Q$. humboldtii se empleó el modelo específico desarrollado por Pérez y Díaz (2010) en bosques andinos de Santander y Cundinamarca. La biomasa abajo del suelo se estimó mediante la ecuación general de Cairns, Brown, Helmer y Baumgardner (1997) para bosques tropicales (Tabla 1). Finalmente, el carbono se estimó empleando el default de fracción de carbono (0.47; IPCC, 2006).

Análisis estadístico: Se empleó el modelo estadístico, Donde; $y$ : observaciones de la unidad experimental; $\mu$ : media general; $S$ : efecto del tratamiento y $E E$ : error experimental. La comparación de medias se realizó mediante la prueba LSD Fisher entre usos del suelo, con un nivel de significancia de 0.05 mediante el programa InfoStaf (Balzarini et al., 2008).

TABLA 1

Modelos alométricos empleados de biomasa aérea ( $\mathrm{Ba})$ y biomasa abajo del suelo $(\mathrm{Br})$ para estimar la biomasa en bosques y pasturas de Santa Isabel, Tolima, Colombia

TABLE 1

Allometric models used for aboveground biomass (Ba) and below-ground biomass ( $\mathrm{Br}$ ) to estimate the biomass in forests and pastures of Santa Isabel, Tolima, Colombia

\begin{tabular}{llcl}
\multicolumn{1}{c}{$\begin{array}{c}\text { Ecosistema/ } \\
\text { zona de vida }\end{array}$} & \multicolumn{1}{c}{ Especie } & \multicolumn{1}{c}{ Modelo } & \multicolumn{1}{c}{ Fuente } \\
Bosque Montano & Multiespecie & $\mathrm{Ba}=\mathrm{e}^{((-1,053+2,079 * \operatorname{Ln}(\mathrm{dap})+\operatorname{Ln}(\mathrm{p})))}$ Álvarez et al. (2012) \\
Bosque Andino & Q. humboldtii & $\mathrm{Ba}=-5,864+0,906 *$ dap $^{2}$ & Pérez \& Díaz (2010) \\
& W. auriculata, Miconia sp. y Baccharis $\mathrm{sp.}$. & $\mathrm{Ba}=\mathrm{e}^{((-1.85+2.11 * \operatorname{Ln}(\mathrm{dap})))}$ & Lerma \& Orjuela (2014) \\
Bosque tropical & Multiespecie & $\mathrm{Br}=\mathrm{e}^{((-1,058+0,88 * \ln (\mathrm{Ba})))}$ & Cairns et al. (1997) \\
\hline
\end{tabular}




\section{RESULTADOS}

Estructura horizontal: El bosque presentó una abundancia de $642 \pm 125$ individuos/ha. Se registraron 321 individuos, pertenecientes a 25 familias, 39 géneros, 43 especies y morfoespecies en 0.5 ha de este sistema de uso del suelo. La especie más abundante fue $Q$. humboldtii (Fagaceae), seguida de Ladenbergia macrocarpa (Rubiaceae) con $210 \pm 92$ y $118 \pm$ 91 individuos/ha, respectivamente (Fig. 1).
Contrastando con estos resultados, en pasturas se encontró una abundancia de $175 \pm 21$ individuos/ha, distribuidos en 8 familias, 9 géneros y 10 especies y morfoespecies que fueron encontrados en 0.35 ha. La especie más abundante en pasturas fue Weinmannia pubescens (Cunoniaceae), seguido de Eugenia sp. y Myrciantes sp., (Myrtaceae), con $43 \pm 23 ; 34 \pm 7$ y $31 \pm$ 9 individuos/ha, respectivamente (Fig. 1). Se encontraron diferencias significativas $(\mathrm{P}<0.05)$

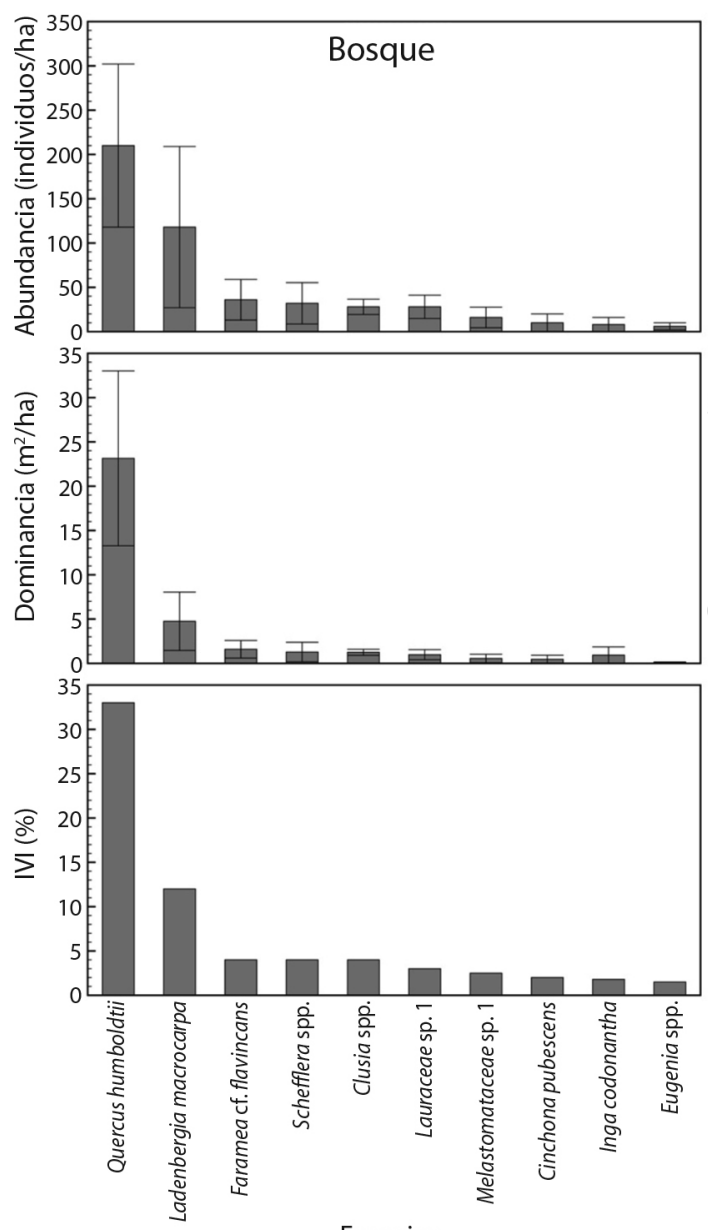

Especies

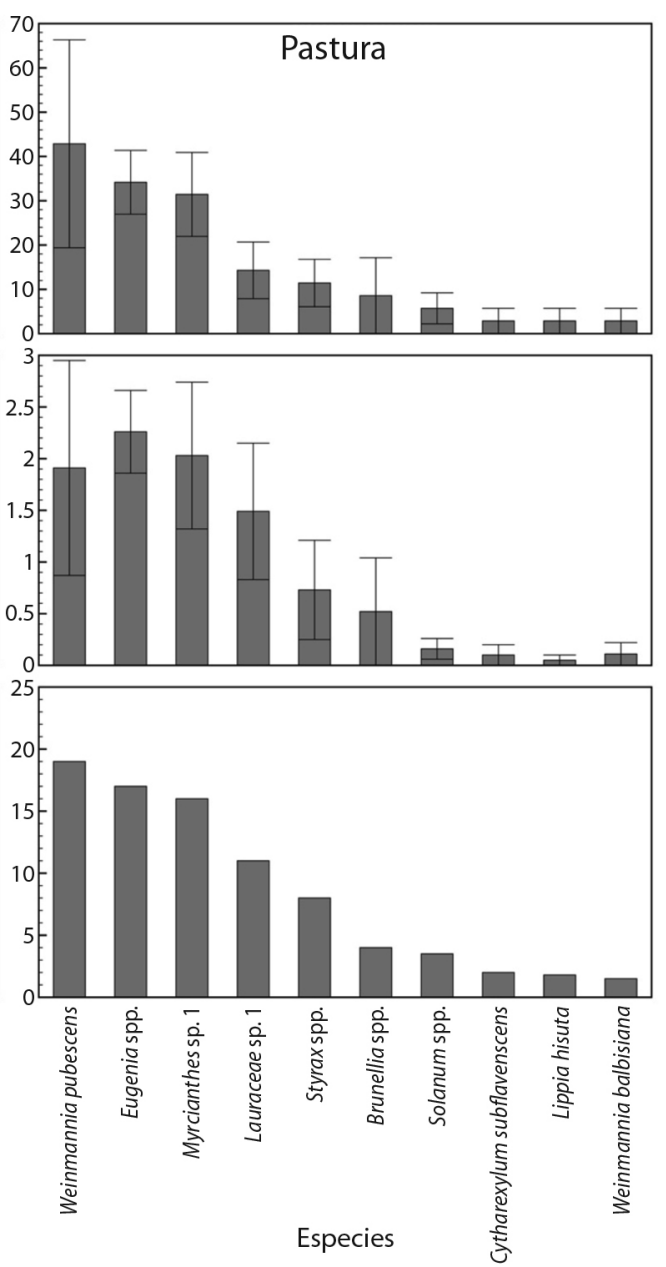

Fig. 1. Abundancia, dominancia e Índice de Valor de Importancia (IVI) de las especies más representativas (dap $\geq 10 \mathrm{~cm}$ ) en bosques primarios intervenidos y pasturas en paisajes altoandinos del municipio de Santa Isabel, Tolima, Colombia. Las barras de error corresponden al error estándar.

Fig. 1. Abundance, dominance and Importance Value Index (IVI) of the most representative species (dap $\geq 10 \mathrm{~cm}$ ) in primary forests intervened and pastures in high Andean landscapes of the municipality of Santa Isabel, Tolima, Colombia. The error bars correspond to the standard error. 
en la abundancia y riqueza entre los dos sistemas de uso del suelo.

Se presentaron diferencias estadísticas ( $\mathrm{P}$ $<0.05$ ) en la dominancia entre bosques y pasturas; sin embargo, estas últimas presentan un valor alto $\left(39.1 \pm 3.7\right.$ vs $\left.10.9 \pm 1.1 \mathrm{~m}^{2} / \mathrm{ha}\right)$. La especie común en estos usos del suelo fue Eugenia sp., con $6 \pm 4$ y $35 \pm 7$ individuos/ha en bosques y pasturas, respectivamente (Fig. 1), la cual fue también dominante en pasturas $\left(2.3 \pm 0.4 \mathrm{~m}^{2} / \mathrm{ha}\right)$; mientras que $Q$. humboldtii fue la más dominante en bosques $(23.1 \pm 9.9$ $\mathrm{m}^{2} /$ ha) (Fig. 1).

Q. humboldtii, fue la especie con mayor importancia ecológica, seguida de L. macrocarpa y Faramea cf. flavincans, presentaron los mayores valores de importancia ecológica $(\mathrm{IVI}=32.5 ; 11.2$ y $4.8 \%$, respectivamente) (Fig. 1). Esto confirma el alto valor del roble en este tipo de ecosistemas (León, Vélez, \& Yepes, 2009). En las pasturas, se encontró que Eugenia sp., W. pubescens y Myrcianthes sp. presentaron la mayor importancia ecológica: IVI de 19.5 , 17.6 y $15.8 \%$, respectivamente (Fig. 1). El índice de Shannon-Wiener muestra que tanto en bosque (1.52 \pm 0.33$)$ como en las pasturas $(1.56 \pm 0.08)$ hay alta heterogeneidad, siendo esta mayor en pasturas. El índice de dominancia Simpson indica que el bosque presenta una dominancia mayor que las pasturas (0.41 vs 0.24$)$ y son más diversos los árboles en pasturas que en bosque $(0.76 \pm 0.02$ vs $0.59 \pm 0.11)$.

Las 43 especies de los bosques presentan distribuciones diamétricas desde $10 \mathrm{~cm}$ hasta más de $90 \mathrm{~cm}$, aunque es dominada por las clases menores (más del $50 \%$ de los individuos con dap entre 10 y $19.9 \mathrm{~cm}$ ), lo cual demuestra su capacidad de regeneración natural. De otro lado, la distribución diamétrica sigue una tendencia de $\mathrm{J}$ invertida, de estructura completa, en donde más del $50 \%$ de los individuos son pequeños $(10>$ dap $>19.9 \mathrm{~cm})$, mientras que los árboles grandes (dap $>60 \mathrm{~cm}$ ) presentan una baja abundancia (Fig. 2). El bosque presentó una estructura disetánea. La abundancia promedio de Q. humboldtii fue de 104 individuos/ ha con dap entre 10 y $105 \mathrm{~cm}$, lo cual coincide con su alta dominancia (Fig. 2). En cambio, los diámetros de las 10 especies de árboles encontrados entre las pasturas, van desde 10 hasta $59.9 \mathrm{~cm}$, sin evidenciar una tendencia de J invertida al haber una disminución de árboles entre los 20 y los $29.9 \mathrm{~cm}$ de dap (Fig. 2).

El almacenamiento de carbono en bosque fue más de cuatro veces superior a la registrada en pasturas, lo cual arroja diferencias estadísticas $(\mathrm{P}>0.05)(125.0 \pm 30.0 \mathrm{vs} 18.4 \pm 1.8 \mathrm{t} / \mathrm{ha}$, respectivamente) (Fig. 2).

\section{DISCUSIÓN}

La abundancia encontrada en bosques andinos naturales intervenidos de Santa Isabel difiere a lo reportado por Campo (2010), quien registró 890 individuos/ha y por Rangel (2011) quien encontró 1580 individuos/ha. Esta diferencia puede atribuirse al devastador impacto que la intervención antrópica está causando en los bosques de Santa Isabel. En otro estudio, en el Santuario de Flora y Fauna de GuanentáAlto Río Fonce, Colombia, se encontró una mayor abundancia (5 760 individuos/ha), pero muestreando árboles con dap $>2.5 \mathrm{~cm}$ (Galindo et al., 2003). En contraste, los estudios de diversidad de árboles en pasturas alto-andinas son escasos; sin embargo, Maclaren, Buckley y Hale (2014) han reportado una riqueza de cerca de seis especies. Esta diversidad permite mitigar la conversión de bosques a pasturas, al albergar una importante riqueza de árboles y otras leñosas perennes, lo cual a su vez genera servicios ecosistémicos como la captura de carbono, el mejoramiento de la fertilidad del suelo, embellecimiento del paisaje, forraje para el ganado, conservación de fuentes hídricas y generación de nichos para el aumento de la biodiversidad (Muñoz et al., 2013).

En Colombia, desde la década de los 80's, Van der Hammer y Cleef (1983) evidenciaron la dominancia de $Q$. humboldtii en sitios con una altitud entre 2000 y $3000 \mathrm{~m}$. Estudios más recientes estiman un $23 \%$ de IVI de esta especie en los bosques altoandinos de Boyacá y Santander, Colombia (Galindo et al., 2003). Así mismo, valores de IVI superiores a los 

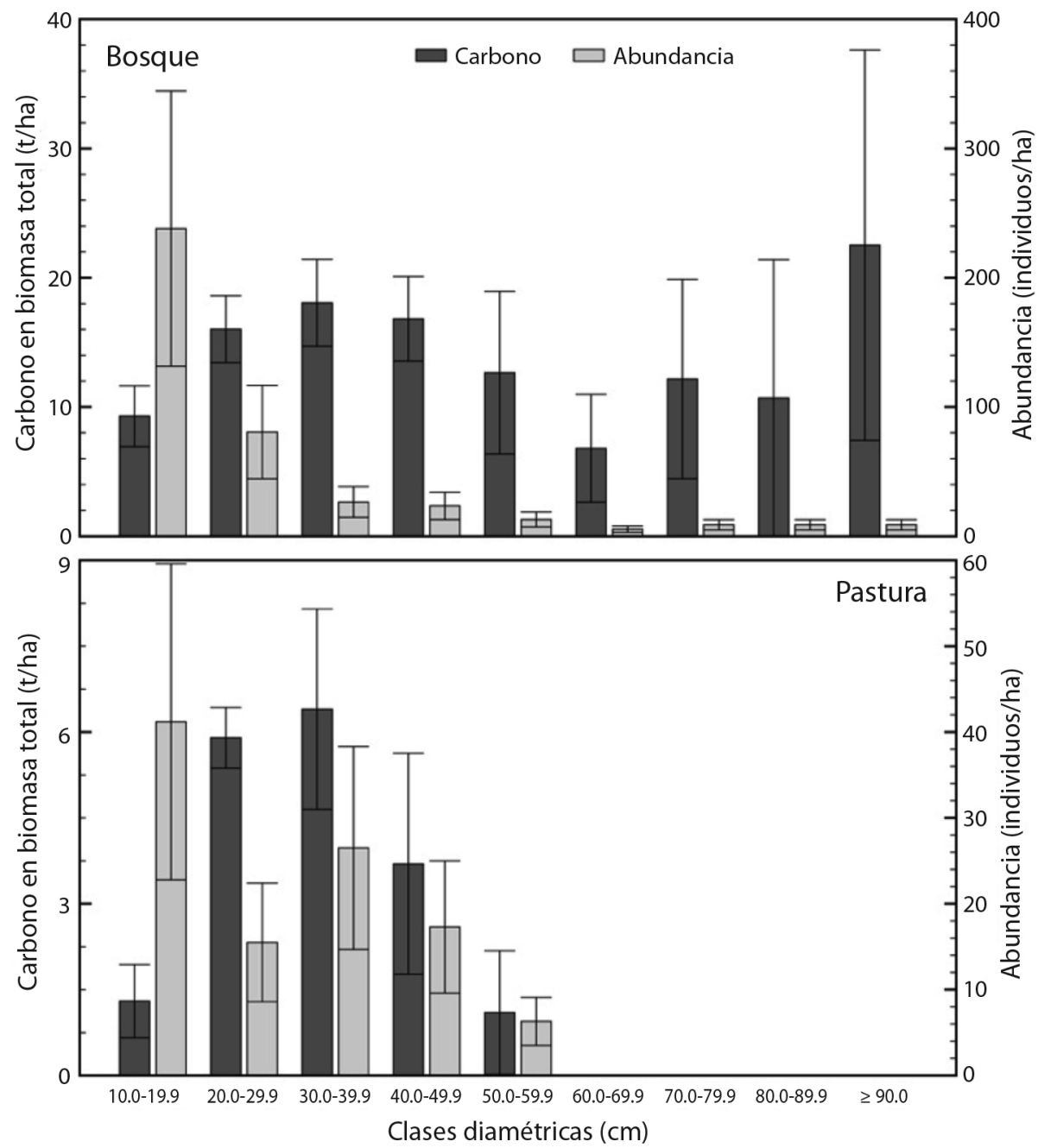

Fig. 2. Distribución diamétrica de árboles con dap $>10 \mathrm{~cm}$ en bosques primarios intervenidos y pasturas en paisajes altoandinos en el municipio de Santa Isabel, Tolima, Colombia. Las barras de error corresponden al error estándar de la media.

Fig. 2. Diametric distribution of trees with $\mathrm{dbh}>10 \mathrm{~cm}$ in primary intervened forests and pastures in high Andean landscapes in the municipality of Santa Isabel, Tolima, Colombia. The error bars correspond to the standard error of the mean.

hallados en este estudio fueron reportados por León y Giraldo (2009) en robledales de Antioquia (entre 44.6 y $77.2 \%$, respectivamente). A pesar de las condiciones de los bosques dominados por $Q$. humboldtii, éstos se encuentran amenazados. Q. humboldtii y Podocaurpus oleifolis han sido categorizadas como vulnerables (VU) en la lista roja de la International Union for Conservation of Nature (IUCN) por su riesgo de extinción y/o grado de deterioro poblacional (Cárdenas \& Salinas, 2006). De la misma forma, el IDEAM (2017a) menciona la drástica aceleración de la deforestación con un incremento del $23 \%$ entre el 2016 y 2017. La superficie boscosa de Colombia muestra una disminución de 5.3 millones de hectáreas entre 1990 y 2015 (WWF-Colombia, 2017).

Los bosques de Santa Isabel presentan una gran ventaja para su dinámica poblacional y conservación, ya que se registraron un área 
basal de $39.1 \pm 3.7 \mathrm{~m}^{2} /$ ha en árboles de varias dimensiones $(10 \mathrm{~cm}>$ dap $>90 \mathrm{~cm})$ (Fig. 2). Esto contrasta con un bosque montano en el sur del Ecuador, donde Aguirre, Reyes, Quizhpe y Cabrera (2017) encontraron una menor dominancia $\left(16.9 \mathrm{~m}^{2} / \mathrm{ha}\right)$, debido principalmente a la mayor abundancia de árboles con menos de $60 \mathrm{~cm}$ de dap y a la diferencia estructural del bosque por estar en recuperación. En este sentido, Moreno y Cuartas (2015) seleccionaron a $Q$. humboldtii, $W$. pubescens y Myrsine Coriacea como las principales especies nativas que promueven el proceso de regeneración en bosques andinos en Antioquia, debido a su alto IVI y alta disponibilidad de semillas. Cortolima (2008) afirma que, para los municipios de Santa Isabel y Anzoátegui, el valor ecológico de $Q$. humboldtii fue $29.2 \%$, el de $W$. pubescens $6.2 \%$, Nectandra sp. $6.1 \%$ y el de Clusia alata $4.5 \%$. Estos valores altos de IVI para esta especie demuestra su importancia ecológica en otras áreas andinas del país y su conservación debería ser dirigida a un área mayor.

Así mismo, la distribución diamétrica es una característica clave de los bosques tropicales y brinda información de la dinámica poblacional, ya que posee una mayor abundancia en las clases inferiores, lo cual garantiza el reclutamiento y crecimiento (Aguirre et al., 2017). Sin embargo, esto no asegura el mantenimiento de los robledales en el tiempo, puesto que las poblaciones están expuestas a la extracción de madera y a la presión de las actividades agropecuarias circundantes (Cortolima-Universidad del Tolima, 2007).

La diversificación de los árboles de especies nativas en pasturas tiene consecuencias sobre la biodiversidad, ya que al ser más diverso un ecosistema, es más productivo y su resiliencia es mayor (Montealegre, Lagos, \& Vélez, 2016) en comparación con las pasturas sin árboles. Por lo cual, las decisiones de los productores son fundamentales para el establecimiento y permanencia de especies arbóreas en las pasturas. En Colombia, son escasos los predios que emplean sistemas agroforestales para mejorar la productividad y generar beneficios ambientales (Uribe et al., 2011). Es necesario educar y brindar apoyo técnico con el fin de incentivar el uso de estas prácticas en el sector agropecuario de país, y así influir positivamente en las características del suelo, del microclima, la hidrología, el almacenamiento de carbono y otros beneficios ambientales de los árboles (Canal \& Andrade, 2019).

En este sentido, el incremento de los árboles en zonas intervenidas es una alternativa práctica para la regulación ambiental, la producción de biomasa y la capacidad de almacenar carbono (Segura, Andrade, \& Mojica, 2019; Canal \& Andrade, 2019) y de otros servicios ecosistémicos como la regulación hídrica (Andrade et al., 2018). La promoción de sistemas agroforestales en la zona andina requiere acciones político-administrativas que incluyan especies funcionalmente ecológicas y de uso múltiple que garantice un aporte a la seguridad alimentaria, al bienestar de la comunidad y se vea reflejado en la economía de la región (Montealegre et al., 2016).

Resultados similares de almacenamiento de carbono han sido reportados por Segura et al. (2019) en bosques nativos en áreas de influencia del páramo de Anaime, Colombia (147 t C/ha). Así mismo, Restrepo, Orrego y Galeano (2012) estimaron 167.9 t C/ha en bosques secundarios del norte de Antioquia. Otros estudios reportan valores inferiores, tal como Andrade et al. (2018) en la cuenca del río Combeima, Ibagué, Colombia $(61.1$ t C/ ha). Por otro lado, Álvarez, Calderón, Krasilnikov y García (2013), encontraron stocks de carbono mucho mayores en bosques montanos de niebla de Oaxaca, México (207.3 t C/ha). El almacenamiento de carbono en la presente investigación se explica por la dominancia de $Q$. humboldtii, que presenta individuos de gran tamaño que contribuyen con más del 50 $\%$ del carbono. Yépes et al. (2015) mencionan también otros factores que pueden explicar la variabilidad del almacenamiento de carbono en los usos del suelo, tal como el clima, la precipitación, tipo de suelo, la pendiente del terreno y la distribución de las especies según el gradiente altitudinal. 
A pesar que las pasturas almacenaron menos carbono que los bosques naturales, los árboles incrementan la captura de GEI, con la consecuente mitigación del cambio climático y permitiendo, a la vez, la producción ganadera (Fig. 2). Jiménez et al. (2019) estimaron en 9.8 t/ha el carbono almacenado en sistemas silvopastoriles de Ecuador. Estos autores evidencian valores menores a los hallazgos del presente estudio debido a que el componente arbóreo presentaba una mayor abundancia (497 individuos/ha) pero con árboles de menor tamaño (3.8 $\mathrm{cm}$ a $30.2 \mathrm{~cm}$ de dap).

En sistemas de pasturas con árboles se ha detectado un rango muy amplio de valores de carbono en biomasa, lo cual depende básicamente de la abundancia y dominancia de arbóreas y de la sombra que éstas proyectan (Andrade, Brook, \& Ibrahim, 2008). Al igual que en los fragmentos de bosque, el mantener e incrementar árboles en pasturas degradadas también promueven servicios de abastecimiento, de apoyo, de regulación y culturales (Andrade et al., 2017). En el territorio colombiano, los sistemas silvopastoriles requieren de acciones de política ambiental, tal como la Política de Crecimiento Verde de Colombia (Howland et al., 2019) y de la planeación y establecimiento de estrategias que contribuyan con la reducción del $30 \%$ de las emisiones de GEI para el 2030 (Ministerio de Ambiente y Desarrollo Sostenible, 2019).

El mantener o establecer árboles en pasturas es una opción para las pasturas degradadas, al permitir la producción y la conservación del carbono ( 18.4 t/ha), convirtiendo en sistemas ganar-ganar. Estos sistemas de producción son estratégicos para el manejo de paisajes agropecuarios, debido a sus beneficios ambientales, tal como la mencionada captura de carbono y aquellos derivados de la conservación de la biodiversidad (Araya \& Carvajal, 2019). Los sistemas silvopastoriles permiten la conectividad de parches de bosque fragmentados y contribuyen a propiciar las interacciones biológicas y el mantenimiento de los servicios ecosistémicos necesarios para el bienestar social y la mitigación del cambio climático
(Pozo, 2019). La cuantificación de la captura de carbono en estos dos sistemas, como servicio ecosistémico, es una estrategia fundamental para la mitigación del cambio climático que requiere de planes de seguimiento, restauración y monitoreo (Jiménez et al., 2019).

Declaración de ética: los autores declaran que todos están de acuerdo con esta publicación y que han hecho aportes que justifican su autoría; que no hay conflicto de interés de ningún tipo; y que han cumplido con todos los requisitos y procedimientos éticos y legales pertinentes. Todas las fuentes de financiamiento se detallan plena y claramente en la sección de agradecimientos. El respectivo documento legal firmado se encuentra en los archivos de la revista.

\section{AGRADECIMIENTOS}

Los autores agradecen a los propietarios de los predios del municipio de Santa Isabel, por permitir el ingreso y la toma de datos para el análisis del presente estudio. A Miguel Acuña, Diana Canal, María del Pilar Marín, María A. Rivera, Sebastián Rojas, Alexandra Huertas, por el apoyo en campo. A la Oficina Central de Investigaciones de la Universidad del Tolima por la financiación del proyecto con código 530114.

\section{RESUMEN}

Introducción: La acelerada transformación de los usos del suelo en los Andes colombianos han afectado la diversidad y la provisión de servicios ecosistémicos. Objetivo: Se caracterizó la estructura y composición florística, junto a la estimación del carbono almacenado en biomasa de bosques primarios intervenidos y pasturas con árboles en Santa Isabel, Tolima. Métodos: En cada sistema, se establecieron cinco parcelas de muestreo: rectangulares de $1000 \mathrm{~m}^{2}$ cada una en bosques y circulares de $707 \mathrm{~m}^{2}$ en pasturas, para medir todos los individuos con diámetro a $1.30 \mathrm{~m}$ de altura, $\geq 10 \mathrm{~cm}$. La biomasa se estimó a partir de modelos alométricos multi-especies y un modelo genérico en el caso de biomasa abajo del suelo. Resultados: En el bosque se registró una abundancia promedio de $642 \pm 125$ individuos/ha, se encontraron 25 familias, 39 géneros, 43 especies y morfoespecies en 0.5 ha de muestreo. Quercus 
humboldtii y Ladenbergia macrocarpa fueron las especies con el mayor Índice de Valor de Importancia (IVI = 32.5 y 11.2, respectivamente) en los bosques. En pasturas se encontraron $175.0 \pm 21.3$ individuos/ha, pertenecientes a 8 familias, 9 géneros, 10 especies y morfoespecies en 0.35 ha de muestreo. Eugenia spp. y Weinmannia pubescens tuvieron la mayor importancia ecológica (19.5 y 17.6 $\%$, respectivamente) en pasturas. El bosque almacenó en promedio $125.0 \pm 30.0 \mathrm{t} \mathrm{C} / \mathrm{ha}$, comparado con 18.4 $\pm 1.8 \mathrm{t} \mathrm{C} /$ ha en pasturas. Conclusiones: Estos hallazgos muestran alta capacidad de almacenamiento de carbono en estos usos del suelo, lo cual contribuye a mantener o disminuir la concentración de gases de efecto invernadero en la atmósfera, y por ende, son un referente que permite desarrollar estrategias de conservación, con miras a mitigar el cambio climático.

Palabras clave: almacenamiento de carbono; biomasa; bosque andino; diversidad; Quercus humboldtii.

\section{REFERENCIAS}

Aguirre, Z., Reyes, B., Quizhpe, W., \& Cabrera, A. (2017). Composición florística, estructura y endemismo del componente leñoso de un bosque montano en el sur del Ecuador. Arnaldoa, 24(2), 543-556.

Álvarez, E., Duque, A., Saldarriaga, J., Cabrera, K., Salas, G., Valle, I.,... Rodríguez, L. (2012). Tree above-ground biomass allometries for carbon stocks estimation in the natural forests of Colombia. Forest Ecology and Management, 267, 297-308.

Álvarez, G., Calderón, N.E., Krasilnikov, P., \& García, F. (2013). Almacenes de carbono en bosques montanos de nieva de la Sierra norte de Oaxaca, México. Agrociencia, 47, 171-180.

Andrade, H.J., Brook, R., \& Ibrahim, M. (2008). Growth, production and carbon sequestration of silvopastoral systems with native timber species in the dry lowlands of Costa Rica. Plant and Soil, 308(1-2), $11-22$.

Andrade, H.J., Segura, M.A., Marín, M.P., Canal, D.S., Sierra, E., Acuña, L.M.,... Izquierdo, O. (2018). Potencial de adaptación al cambio climático de sistemas de uso del suelo en la cuenca media de río Combeima (Ibagué, Colombia). En H. Andrade (Ed.), Servicios ecosistémicos aportados por sistemas de producción en laderas de la cuenca media del rio Combeima (Departamento del Tolima, Colombia): un aporte a la gestión del recurso hídrico (pp. 129148). Ibagué, Tolima: Sello Editorial Universidad del Tolima.

Andrade, H.J., Segura, M.A., \& Sierra, E. (2017). Percepción local de los servicios ecosistémicos ofertados en fincas agropecuarias de la zona seca del norte del Tolima, Colombia. Luna Azul, 45, 42-58.
Araya, O., \& Carvajal, J.P. (2019). Composición y riqueza de avifauna en cuatro tipos de cobertura vegetal de San Ramón, Costa Rica. Cuadernos de Investigación, 11(2), 154-161.

Avella, A., \& Cárdenas, L.M. (2010). Conservación y uso sostenible de los bosques de roble en el corredor de conservación Guantiva-La Rusia-Iguaque, Departamentos de Santander y Boyacá, Colombia. Colombia Forestal, 13(1), 5-25.

Balzarini, M.G., Gonzalez, L., Tablada, M., Casanoves, F., Di Rienzo, J.A., \& Robledo, C.W. (2008). Software estadístico InfoStat: Manual del usuario. Córdoba, Argentina: Editorial Brujas.

Cairns, M.A., Brown, S., Helmer, E.H., \& Baumgardner, G.A. (1997). Root biomass allocation in the world's upland forests. Oecologia, 111, 1-11.

Campo, J.M. (2010). Estructura, riqueza y composición de plantas arboresentes en un bosque de niebla entresacado del Tolima (Colombia). Acta Biológica Colombiana, 15(2), 247-262.

Canal, D.S., \& Andrade, C.H.J. (2019). Sinergias mitigación - adaptación al cambio climático en sistemas de producción de café (Coffea arabica), de Tolima, Colombia. Revista de Biología Tropical, 67(1), 36-46.

Cárdenas, D., \& Salinas, N. (2006). Libro Rojo de plantas de Colombia. Especies maderables amenazadas. Bogotá, Colombia: Instituto Amazónico de Investigaciones Científicas SINCHI-Ministerio de Ambiente, Vivienda y Desarrollo Territorial.

Consejo Municipal de Santa Isabel. (2016). Acuerdo municipal N. 004. 31 de Mayo de 2016. Santa Isabel, Tolima.

Cortolima (2008). Plan de ordenación y manejo de la Cuenca hidrográfica mayor del rio Totare convenio Cortolima-Corpoica-Sena-Universidad del Tolima. Recuperado de https:/www.cortolima.gov.co/ sites/default/files/images/stories/centro_documentos/ pom_totare/diagnostico/kj_210111_flora.pdf

Cortolima-Universidad del Tolima. (2007). Plan General de Ordenamiento Forestal Para el Departamento del Tolima. Recuperado de https://www.cortolima.gov. co/sites/default/files/images/stories/centro_documentos/estudios/tomo_01.pdf

DANE. (2005). Estimación y proyección de población nacional, departamental y municipal total por área 1985-2020. Bogotá, Colombia. Recuperado de https://www.dane.gov.co/files/investigaciones/poblacion/proyepobla06_20/Municipal_area_1985-2020. xls

DANE. (2014). Censo nacional agropecuario Colombia. Bogotá, Colombia. Recuperado de https://www.dane. gov.co/files/CensoAgropecuario/avanceCNA/CNA_ agosto_2015_new_present.pdf 
FAO. (1999). Educación ambiental para el trópico de Cochabamba. Cochabamba, Bolivia. Recuperado de http://www.fao.org/3/ah648s/AH648S00.htm

FAO. (2010). Evaluación de los recursos forestales mundiales. Términos y definiciones. Departamento Forestal, FAO, Roma. Recuperado de http://www. fao.org/3/i1757s/i1757s.pdf

Fernández, F., Bernate, J., \& Melo, O. (2013). Diversidad arbórea y prioridades de conservación de los bosques secos tropicales del sur del departamento del Tolima en el Valle del río Magdalena, Colombia. Actualidades Biológicas, 35(99), 161-183.

Galindo, R., Betancur, J., \& Cadena, J. (2003). Estructura y composición florística de cuatro bosques andinos del Santuario de Flora y Fauna Guanentá-Alto Río Fonce, cordillera oriental colombiana. Caldasia, 25(2), 313-335.

Hammer, O., Harper, T., \& Ryan, P.D. (2007). PASTPaleontological Statistics software package (Versión 3.16). Retrieved from https://folk.uio.no/ohammer/ past

Holdridge, L.R. (1978). Ecología Basada en Zonas de Vida. San José, Costa Rica: Educativos IICA.

Howland, F., Le, J.F., Martínez, D., Tapasco, J., Loboguerrero, A.M., Sandoval, J.M., ... Villamil, M. (2019). Hacia una política de crecimiento verde para el sector agropecuario en Colombia. Climate Change, Agriculture and Food Security, 1-6.

IDEAM. (2016). Inventario nacional y departamental de Gases Efecto Invernadero-Colombia. Tercera Comunicación Nacional de Cambio Climático. Bogotá, Colombia: IDEAM, PNUD, MADS, DNP, CANCILLERÍA, FMAM

IDEAM. (2017a). Resultados monitoreo de la deforestación. Recuperado de http://www. ideam.gov.co/documents/24277/72115631/ A c tualizacion cifras $2017+$ F I N A L . pdf/40bc4bb3-370c-46̈39-91ee-e4c6cea97a07

IDEAM. (2017b). Banco de datos de estaciones meteorológicas en Colombia. Instituto de Hidrología, Meteorología y Estudios Ambientales-IDEAM. Recuperado de atenciónalciudadano@ideam.gov.co

IPCC. (2006). IPCC Guidelines for National Greenhouse Gas Inventories. H.S. Eggleston, L. Buendia, K. Miwa, T. Ngara \& K. Tanabe (Eds.). Tokyo, Japan: IGES

Jiménez, E.R., Fonseca, W., \& Pazmiño, L. (2019). Sistemas silvopastoriles y cambio climático: estimación y predicción de biomasa arbórea. La Granja: Revista de Ciencias de la Vida, 29(1), 45-55.

León, J.D., Vélez, G., \& Yepes, A.P. (2009). Estructura y composición florística de tres robledales en la región norte de la cordillera central de Colombia. Revista de Biología Tropical, 57(4), 1165-1182.

León, J.D., \& Giraldo, E. (2009). Crecimiento diamétrico en robledales del norte y centro de Antioquia, Colombia. Crónica Forestal y del Medio Ambiente, 15(1), 119-138

Lerma, M.A., \& Orjuela, E.L. (2014). Modelos alométricos para la estimación de la biomasa aérea total en el páramo de Anaime, Departamento del Tolima, Colombia (Trabajo de grado). Universidad del Tolima.

Maclaren, C., Buckley, H.L., \& Hale, R.J. (2014). Conservation of forest biodiversity and ecosystem properties in a pastoral landscale of the Ecuadorian Andes. Agroforestry Systems, 88, 369-381.

Martínez, A., Cadahía, E., Fernández, B., Gutiérrez, G., Nevares, I., \& Álamo, M. (2017). Quercus humboldtii (Colombian oak): Characterisatión of Wood phenolic composition with respect to traditional oak wood used in oenology. Ciência Técnica Vitinícola, 32(2), 93-101.

Mathez, S.L., Peralvo, M., \& Báez, S. (2017). Hacia la conservación y la gobernanza sostenible de los paisajes de bosques andinos: una agenda de investigación. Quito, Ecuador: Programa Bosques Andinos de la Agencia Suiza para el Desarrollo y la CooperaciónCOSUDE, CONDESAN, Helvetas Swiss Intercooperation, CDE - University of Bern.

Ministerio de Ambiente, Vivienda y Desarrollo Territorial. (2006). Resolución Número 096 del 20 de Enero de 2006. Bogotá D.C. Colombia. Recuperado de https:// icbf.gov.co/cargues/avance/docs/resolucion_minambientevdt 0096 2006.htm

Ministerio de Ambiente y Desarrollo Sostenible. (2019). Colombia se propone aumentar a $30 \%$ la meta de reducción de emisiones de GEI para 2030. MinAmbiente, Bogotá D.C. Colombia. Recuperado de https://www.minambiente.gov.co/index.php/ noticias-minambiente/2480-colombia-se-proponeaumentar-a-30-la-meta-de-reduccion-de-emisionesde-gei-para-2030

Montealegre, M., Lagos, T., \& Vélez, J. (2016). Agrodiversidad de los huertos caseros de la región andina del sur de Colombia. Revista de Ciencias Agrícolas, 34(1), 50-63.

Moreno, C. (2001). Métodos para medir la biodiversidad. Zaragoza, España: M\&T-Manuales y Tesis SEA.

Moreno, C.E., Barragán, F., Pineda, E., \& Pavón, N.P. (2011). Reanálisis de la diversidad alfa: alternativas para interpretar y comparar información sobre comunidades ecológicas. Revista Mexicana de Biodiversidad, 82, 1249-1261. 
Moreno, D.J., \& Cuartas, S.E. (2015). Sobrevivencia y crecimiento de plántulas de tres especies arbóreas en áreas de bosque montano andino degradadas por ganadería en Colombia. Acta Biológica Colombiana, 20(2), 85-100.

Muñoz, D.A., Calvache, D.A., \& Yela, J.F. (2013). Especies forestales con potencial agroforestal para las zonas altas en el departamento de Nariño. Revista de Ciencias Agrícolas, 29(1), 38-53.

Pérez, M.C., \& Díaz, J.J. (2010). Estimación del carbono contenido en la biomasa forestal aérea de dos bosques andinos en los departamentos de Santander y Cundinamarca (Tesis de grado). Universidad Distrital Francisco José de Caldas, Bogotá, Colombia.

Potosí, A., Villalba, J.C. \& Arboleda, L.Y. (2017). Productos forestales no maderables asociados a bosques de roble Quercus humboldtii bonpl en la Vega, Cauca. Biotecnología en el Sector Agropecuario y Agroindustrial, 15(2), 22-29.

Pozo, P.P. (2019). Los sistemas silvopastoriles. Una alternativa para el manejo ecológico de los pastizales: experiencias de su aplicación en Cuba. Cadernos de Agroecologia, 14(2), 1-7.

Pujade, J., Rodríguez, P.A., \& Caicedo, G. (2015). Dos nuevas especies de Zapatella (Hym., Cynipidae) para Colombia que producen agallas en ramas de Quercus humboldtii (Fagaceae). Butlleti ICHN, 79, 79-90.

Rangel, J.O. (2011). Colombia Diversidad biológica XI. Patrones de la estructura y la riqueza de la vegetación en Colombia. Bogotá, Colombia: Universidad Nacional de Colombia.
Restrepo, H.I., Orrego, S.A., \& Galeano, O.J. (2012). Estructura de bosques secundarios y rastrojos montano bajos del norte de Antioquia, Colombia. Colombia Forestal, 15(2), 173-189.

Rojas, A.S., Andrade, H.J., \& Segura, M.A. (2018). Los suelos del paisaje alto-andino de Santa Isabel (Tolima, Colombia) ¿Son sumideros de carbono orgánico? Revista U.D.C.A Actualidad \& Divulgación Cientifica, 21(1), 51-59.

Segura, M., Andrade, H., \& Mojica, C. (2019). Floristic structure and composition and carbon storage in native forest of paramo of Anaime, Tolima, Colombia. Ciência Florestal, 29(1), 157-168.

Uribe, F., Zuluaga, A.F., Valencia, L., Murgueitio, E., Zapata, A., Solarte, L... Soto, R. (2011). Establecimiento $y$ manejo de sistemas silvopastoriles. Manual 1, Proyecto Ganadería Colombiana Sostenible. Bogotá, Colombia: GEF.

Van der Hammen, T., \& Cleef, A. (1983). Datos para la historia de la flora Andina. Revista Chilena de Historia Natural, 56, 97-107.

Yépes, A., Herrera, J., Phillips, J., Cabrera, E., Galindo, G., Granados, E., ... Cardona, M. (2015). Contribución de los bosques tropicales de montaña en el almacenamiento de carbono en Colombia. Revista de Biología Tropical, 63(1), 69-82.

WWF-Colombia. (2017). Colombia Viva: un país megadiverso de cara al futuro. Cali, Colombia: WWF-Colombia. 